\title{
Prevalence of institutional delivery and its correlates amongst women of reproductive age in Mozambique: a cross-sectional analysis
}

Sanni Yaya ${ }^{1,2^{*}+}$ D, Dina Idriss-Wheeler ${ }^{3}$, Gebretsadik Shibre $^{4}$, Agbessi Amouzou ${ }^{5}$ and Ghose Bishwajit ${ }^{1+}$

\begin{abstract}
Background: The healthcare system in Mozambique is striving to reduce the high maternal and child mortality rates and stay on par with the Sustainable Development Goals (SDG 3.1). A key strategy to curb maternal and child mortality is to promote the use of professional childbirth services proven to be highly effective in averting maternal deaths. Currently, little is known about the use of childbirth services in Mozambique. The present study investigated the prevalence of professional healthcare delivery services and identified their sociodemographic correlates.

Methods: This study used cross-sectional data on 7080 women aged 15-49years who reported having a child during the past 5 years. The data were collected from the 2011Mozambique Demographic and Health Survey. The outcome variables were the choice of childbirth services that included 1) place of delivery (respondent's home versus health facility), and mode of delivery (caesarean section versus vaginal birth). Data were analyzed using descriptive and multivariate regression methods.

Results: The prevalence of health facility and C-section delivery was 70.7 and 5.6\%, respectively. There was a difference in the use of professional birthing services between urban and rural areas. Having better educational status and living in households of higher wealth quintiles showed a positive association with the use of facility delivery services among both urban and rural residents. Regarding ethnicity, women of Portugais [2.688,1.540,4.692], Cindau [1.876,1.423,2.474] and Xichangana [1.557,1.215,1.996] had relatively higher odds of using facility delivery services than others. Antenatal care (ANC) visits were a significant predictor of facility delivery services both in urban $[\mathrm{OR}=1.655,95 \% \mathrm{Cl}=1.235,2.218]$ and rural $[\mathrm{OR}=1.265,95 \% \mathrm{Cl}=1.108,1.445]$ areas. Among rural women, ANC visit was a significant predictor of C-section delivery [1.570,1.042,2.365].
\end{abstract}

Conclusion: More than a quarter of the women in Mozambique were not using health facility delivery services, with the prevalence being noticeably lower in the rural areas.

Keywords: C-section, Facility delivery, Mozambique, Global health, Women's health, demographic and health surveys

\footnotetext{
* Correspondence: sanni.yaya@uOttawa.ca

Sanni Yaya and Ghose Bishwajit are first joint authors

${ }^{1}$ School of International Development and Global Studies, Faculty of Social

Sciences, University of Ottawa, 120 University Private, Ottawa, ON K1N 6N5,

Canada

${ }^{2}$ The George Institute for Global Health, University of Oxford, Oxford, UK

Full list of author information is available at the end of the article
}

(c) The Author(s). 2020 Open Access This article is licensed under a Creative Commons Attribution 4.0 International License, which permits use, sharing, adaptation, distribution and reproduction in any medium or format, as long as you give appropriate credit to the original author(s) and the source, provide a link to the Creative Commons licence, and indicate if changes were made. The images or other third party material in this article are included in the article's Creative Commons licence, unless indicated otherwise in a credit line to the material. If material is not included in the article's Creative Commons licence and your intended use is not permitted by statutory regulation or exceeds the permitted use, you will need to obtain permission directly from the copyright holder. To view a copy of this licence, visit http://creativecommons.org/licenses/by/4.0/ The Creative Commons Public Domain Dedication waiver (http://creativecommons.org/publicdomain/zero/1.0/) applies to the data made available in this article, unless otherwise stated in a credit line to the data. 


\section{Plain English summary}

The Republic of Mozambique is one of the least developed countries in Southern Africa with a large portion of the population living below the poverty line. Characterized by high fertility rates and plagued by high maternal and child mortality rates, preventative maternity services are underutilized or may be inaccessible. Approximately $30 \%$ of births in the region are attended by family members while $23-40 \%$ are attended by insufficiently trained traditional birth attendants. Access to skilled birth assistance and health facility delivery services requires financial resources and many women in Mozambique cannot afford necessary expensive procedures and services.

Currently, little is known about the use of childbirth services in Mozambique. Using the well-established Andersen's Behavioural Model of Health Service, the present study investigated maternal healthcare service utilization and identified correlated sociodemographic variables. The study used the 2011 Mozambique Demographic and Health Survey cross-sectional data on 7080 women aged 15-49 years who reported having a child during the past 5 yrs.

Findings revealed more than a quarter of women in Mozambique were not using health facility delivery services, particularly in the rural areas. Women with higher educational status and those living in more affluent households were more likely to use health facility delivery services in both rural and urban regions. Furthermore, antenatal care (ANC) visits were a predictor of Csection delivery in rural regions.

\section{Background}

The Republic of Mozambique is located in Southern Africa and is one of the least developed countries in the region $[1,2]$. Since the end of the prolonged civil war in 1992 (1977-1992), the country has introduced a series of macroeconomic reforms to revitalize the economy and initiatives to improve the living standards of the population [3]. Despite the noticeable progress made in the areas of poverty reduction, a large proportion of the population continues to live below the poverty line and have significant challenges in securing basic amenities such as ensuring food security and accessing healthcare [4]. Higher fertility rates (5.24 birth per woman as of 2016), a predominantly rural distribution of the population (67.49 as of 2016), a relatively young age structure (45.2\% under age 15$)$, low life expectancy (59.31 years as of 2017), and high maternal and child mortality rates characterize the demography of Mozambique [5].

According to available estimates, the under-five mortality rate was 72 (per 1000 live births as of 2017) and that of maternal mortality was 480 (per 100,000 live births as of 2015) [1], down from 1500 in 2000; one of the highest rates globally [6]. Approximately 30\% of births in sub-Saharan Africa are unattended or only attended by family members while about $23-40 \%$ are attended by traditional birth attendants (TBAs) [7]. Every day, globally, approximately 830 women die from pregnancy and childbirth-related complications [8]. These deaths, almost all of which take place in lowincome countries, could have been averted through the use of quality obstetric services $[9,10]$. In addition to the lives lost and the emotional distress caused by maternal or neonatal bereavement [11], there is a strong human rights component to maternal child mortality (MCM) which is shaping women's reproductive health policy making mechanisms $[12,13]$.

Reproductive health services in Mozambique are inadequate to meet the needs of a growing population. This is particularly the case for services such as the availability of skilled birth assistance as well as equipment for providing sophisticated procedures such as $\mathrm{C}$-sections $[14,15]$ which are expensive and lead to a significant financial burden for the mother and her family, especially in low-income settings like Mozambique where health insurance coverage is very low [16]. Preferences and utilization of healthcare services are inherently subjective and multifaced, and can themselves be influenced by a host of demographic, sociocultural, environmental, and economic determinants [17-21]. From previous studies, healthcare utilization can be conceptualized as an outcome of proximal and distal factors and their interplay shapes people's perception of health and motivations for action. Understanding these determinants are necessary for making concrete and evidence-driven policy approaches to tackle maternal mortality in Mozambique. We undertook the present study to analyze open-access and nationally-representative data from Mozambique Demographic and Health Survey (DHS 2011). These findings will help advance the understanding of the sociodemographic inequalities in the uptake of skilled birth attendants (SBAs) and C-section services in Mozambique as well as in the neighbouring countries with similar economic and sociocultural environments.

\section{Methodology}

\section{Data source}

Data for this study were collected from the sixth round of Mozambique Demographic and Health Survey. The survey was conducted by the National Statistical Institute (Instituto Nacional de Estatística) and the Ministry of Health (MISAU). The work was finally supported by United States Agency for International Development of the United America (USAID) with Inner City Fund (ICF) International providing technical assistance. Sample population included eligible men ( $15-54$ years) and women (15-49 years) residing in households in urban 
and rural areas, excluding institutions such as hospitals, hotels, dorms. Data collection was done through direct interviews using a tablet-type computer (ComputerAssisted Personal Interview) system and this process lasted from June 2011 to November 2011. Sampling was done using multistage cluster technique which involves stratifying the provinces into primary sampling units (PSUs), and then selecting of households each PSUs. Of the 13,964 households initially selected, a total of 13,718 women were finally interviewed, resulting in a $99 \%$ response rate. These details are available from the final report of Mozambique 2011 DHS and available here: https://dhsprogram.com/publications/publicationFR266-DHS-Final-Reports.cfm.

\section{Outcome measures}

The outcome variables of interest were: 1) place of delivery: home versus health facility, 2) use of c-section: yes versus no.

\section{Explanatory variables}

The selection of explanatory variables was guided by Andersen's Behavioural Model of Health Service utilization which postulates that healthcare utilization is a function of three major factors: 1) predisposing factors, 2) enabling factors and 3) need factors [22]. For this study, the data were secondary and hence the selection of the explanatory variables in line with the behavioral model was not completely possible. Based on the availability in the dataset, the following are included in the analysis: Age (15-19, 20-24, 25-29, 30-34, 35-39, 4044, 45-49); Residency (Urban, Rural); Education (No Education, Primary, Secondary, Higher); Husband's education (No Education, Primary, Secondary, Higher); Occupation (Not Working, Professional/Technical/ Managerial, Agricultural - Self Employed); Wealth quintile (Poorest, Poorer, Middle, Richer, Richest); Electronic Media Access (No, Yes); Heard of Family Planning (FP) on the internet (No, Yes); Religion (Islam, Other); Ethnicity (Emakhuwa, Portugais, Xichangana, Cisena, Elomwe, Cindau, Xitswa, Other); Parity $(1-5,>5)$; Sex of Household Head (Male, Female); Last Child Wanted (Wanted Then, Wanted No More); Place of Delivery (Home, Health facility).

\section{Data analysis}

Data were analyzed with Stata version 14. Dataset was cleaned by applying the inclusion criteria: experience of at least 1 childbirth in the preceding 5 years. As the surveys used cluster sampling techniques, all analyses were adjusted for this by using the svy command [23]. This command uses the information on sampling weight, strata, and primary sampling unit provided with the datasets. Sample characteristics were described as frequencies and percentages. Prevalence of using facility delivery and C-section (for total, urban and rural sample) was presented as bar charts. The predictors of facility delivery and C-section were measured using multivariate analysis. As both of the variables were dichotomous, we used binary logistic regression models and the results expressed using odds ratios (OR) with 95\% confidence intervals (CIs). Each of the outcome variables was analyzed separately for the pooled, urban and rural participants. Model fit statistics were run after the regression analyses using the variance inflation factor (VIF) command. No multi-collinearity was detected as VIF values were below 10 for all the models. All tests were two-tailed and were considered significant at alpha value of $5 \%$.

\section{Results \\ Sample description}

The basic characteristics of the sample population were shown in Table 1. A greater proportion of the participants: were aged $20-29$ years $(48.98 \%)$, from rural residences (63.16\%), had primary education (51.50\%), had no employment (53.59\%), from households with highest wealth quintile $(25.92 \%)$, had access to electronic media $(71.69 \%)$, were followers of Islam $(71.12 \%)$, were of Xichanga ethnicity (19.66\%), had 1-5 children (81.78\%), were from male-headed households (64.90\%), wanted the last child (79.86\%), and received at least four ANC visits (70.75\%).

Figure 1 shows that over three-fifth (70.7\%) of the participants had their last childbirth at a health facility and $29.3 \%$ in their home. The percentage of home delivery was four times as high in the rural areas compared with urban areas $(p<0.05)$.

Figure 2 shows that only $5.6 \%$ of the participants had their last childbirth using c-section. Similar to health facility delivery, the percentage of $\mathrm{C}$-sections performed was markedly higher in the urban areas $(10.2 \%)$ compared with rural areas $(2.9 \%)$.

The predictors of using facility delivery and c-sections were presented in Table 2 and Table 3, respectively. In general, the odds of using facility delivery was lower among women in higher age groups. However, the difference was significant among those aged 40-44 years and in rural areas only. Rural residents had significantly lower odds of using facility delivery $[\mathrm{OR}=0.527$, $95 \% \mathrm{CI}=0.440,0.630]$. Having primary, secondary, and higher education showed a positive association with the use of facility delivery services among both urban and rural residents. In the pooled sample, the odds of using facility delivery service were: primary $[O R=1.737$, $95 \% \mathrm{CI}=1.370,2.203]$, secondary $[\mathrm{OR}=2.608,95 \% \mathrm{CI}=$ $1.840,3.697$, and higher [OR $=1.266,1.012,1.584]$. 
Table 1 Sample characteristics $(n=7080)$

\begin{tabular}{|c|c|c|}
\hline & Freq. & Percent \\
\hline \multicolumn{3}{|l|}{ Age } \\
\hline $15-19$ & 813 & 11.48 \\
\hline $20-24$ & 1771 & 25.01 \\
\hline $25-29$ & 1697 & 23.97 \\
\hline $30-34$ & 1319 & 18.63 \\
\hline $35-39$ & 935 & 13.21 \\
\hline $40-44$ & 394 & 5.56 \\
\hline $45-49$ & 151 & 2.13 \\
\hline Residency & Freq. & Percent \\
\hline Urban & 2608 & 36.84 \\
\hline Rural & 4472 & 63.16 \\
\hline Education & Freq. & Percent \\
\hline No Education & 2139 & 30.21 \\
\hline Primary & 3646 & 51.50 \\
\hline Secondary & 1,22 & 17.23 \\
\hline Higher & 75 & 1.06 \\
\hline Husband's education & Freq. & Percent \\
\hline No Education & 1731 & 26.36 \\
\hline Primary & 3272 & 49.82 \\
\hline Secondary & 1422 & 21.65 \\
\hline Higher & 143 & 2.18 \\
\hline Occupation & Freq. & Percent \\
\hline Not Working & 3794 & 53.59 \\
\hline Professional/Technical/Managerial & 1269 & 17.92 \\
\hline Agricultural - Self Employed & 2017 & 28.49 \\
\hline Wealth index & Freq. & Percent \\
\hline Poorest & 1067 & 15.07 \\
\hline Poorer & 1194 & 16.86 \\
\hline Middle & 1328 & 18.76 \\
\hline Richer & 1656 & 23.39 \\
\hline Richest & 1835 & 25.92 \\
\hline Electronic Media access & Freq. & Percent \\
\hline No & 2004 & 28.31 \\
\hline Yes & 5076 & 71.69 \\
\hline Religion & Freq. & Percent \\
\hline Islam & 5035 & 71.12 \\
\hline Other & 2045 & 28.88 \\
\hline Ethnicity & Freq. & Percent \\
\hline Emakhuwa & 1241 & 17.53 \\
\hline Portugais & 607 & 8.57 \\
\hline Xichangana & 1392 & 19.66 \\
\hline Cisena & 676 & 9.55 \\
\hline Elomwe & 266 & 3.76 \\
\hline Cindau & 426 & 6.02 \\
\hline
\end{tabular}

Table 1 Sample characteristics ( $n=7080)$ (Continued)

\begin{tabular}{cll}
\hline & Freq. & Percent \\
\hline Xitswa & 368 & 5.20 \\
Other & 2104 & 29.72 \\
Parity & Freq. & Percent \\
$1-5$ & 5,79 & 81.78 \\
$>5$ & 1,29 & 18.22 \\
Sex of household head & Freq. & Percent \\
Male & 4595 & 64.90 \\
Female & 2485 & 35.00 \\
Last child wanted & Freq. & Percent \\
Wanted Then & 5654 & 79.86 \\
Wanted No More & 1426 & 20.14 \\
ANC visits & Freq. & Percent \\
$<4$ & 2071 & 29.25 \\
4 or more & 5009 & 70.75 \\
\hline
\end{tabular}

Similar results were observed for husband's education as well, however the odds were significant only among rural women and for secondary $[\mathrm{OR}=1.762,95 \% \mathrm{CI}=$ $1.328,2.338]$ and higher education only $[\mathrm{OR}=1.236$, $95 \% \mathrm{CI}=1.031,1.481]$. Women from the middle [OR $=$ $1.788,95 \% \mathrm{CI}=1.495,2.138]$, richer $[\mathrm{OR}=2.718,95 \% \mathrm{CI}=$ $2.217,3.333]$ and richest $[\mathrm{OR}=4.898,95 \% \mathrm{CI}=3.547$, 6.764] wealth quintile households had significantly higher odds of using facility delivery compared with those in the lowest quintile. Women of Portugais [OR = 2.688, $95 \% \mathrm{CI}=1.540,4.692], \quad$ Cindau $\quad[\mathrm{OR}=1.876$, $95 \% \mathrm{CI}=1.423,2.474]$ and Xichangana $[\mathrm{OR}=1.557,1.215$, 1.996] ethnicity had relatively higher odds of using facility delivery services than others. Rural women in the female headed households had higher odds of using facility delivery services $[\mathrm{OR}=1.184,95 \% \mathrm{CI}=1.022$, 1.371]. Using adequate ANC visits was a significant predictor of facility delivery services both in urban [OR = $1.655,95 \% \mathrm{CI}=1.235,2.218]$ and rural $[\mathrm{OR}=1.265$, $95 \% \mathrm{CI}=1.108,1.445]$ areas.

Regarding the use of c-section services, the most notable predictors were similar to that of facility delivery: rural residence, higher education, higher wealth, and adequate ANC visits. Having higher education showed positive association with the use of c-section among urban $[\mathrm{OR}=3.269,95 \% \mathrm{CI}=1.327,8.050]$ and rural residents with secondary education $[\mathrm{OR}=2.979,1.425$, 6.226]. Cindau women in the urban areas $[\mathrm{OR}=2.669$, $95 \% \mathrm{CI}=1.048,6.801]$ had higher odds of using c-section. Among rural women, using adequate ANC visits was a significant predictor of choosing $\mathrm{c}$-section delivery [OR = $1.570,95 \% \mathrm{CI}=1.042,2.365]$. 

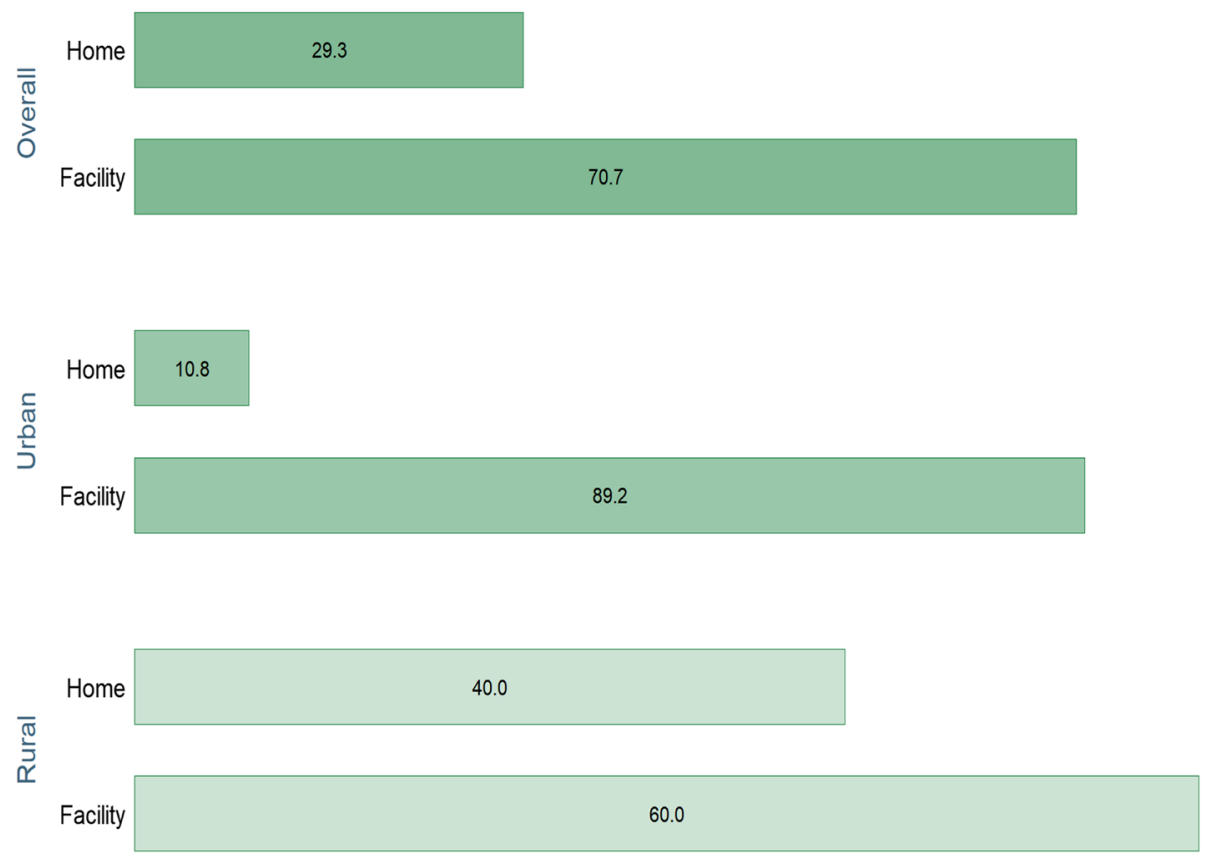

Fig. 1 Prevalence of health facility delivery

\section{Discussion}

Using the data from Mozambique Demographic and Health Survey, findings from this study revealed that little less than one-third of the women in Mozambique were not using health facility delivery services. In the region-specific analysis, the disparity was more evident among rural women, with the prevalence being two-fifth compared with about $90 \%$ among urban women. Urbanrural disparity in the use of maternal and reproductive services is common in Sub-Saharan African countries [24-28]. The growing urban-rural inequality in terms of using lifesaving services poses a major challenge for meeting the goals of reducing maternal and child mortality and morbidity [25]. Stark inequality was observed
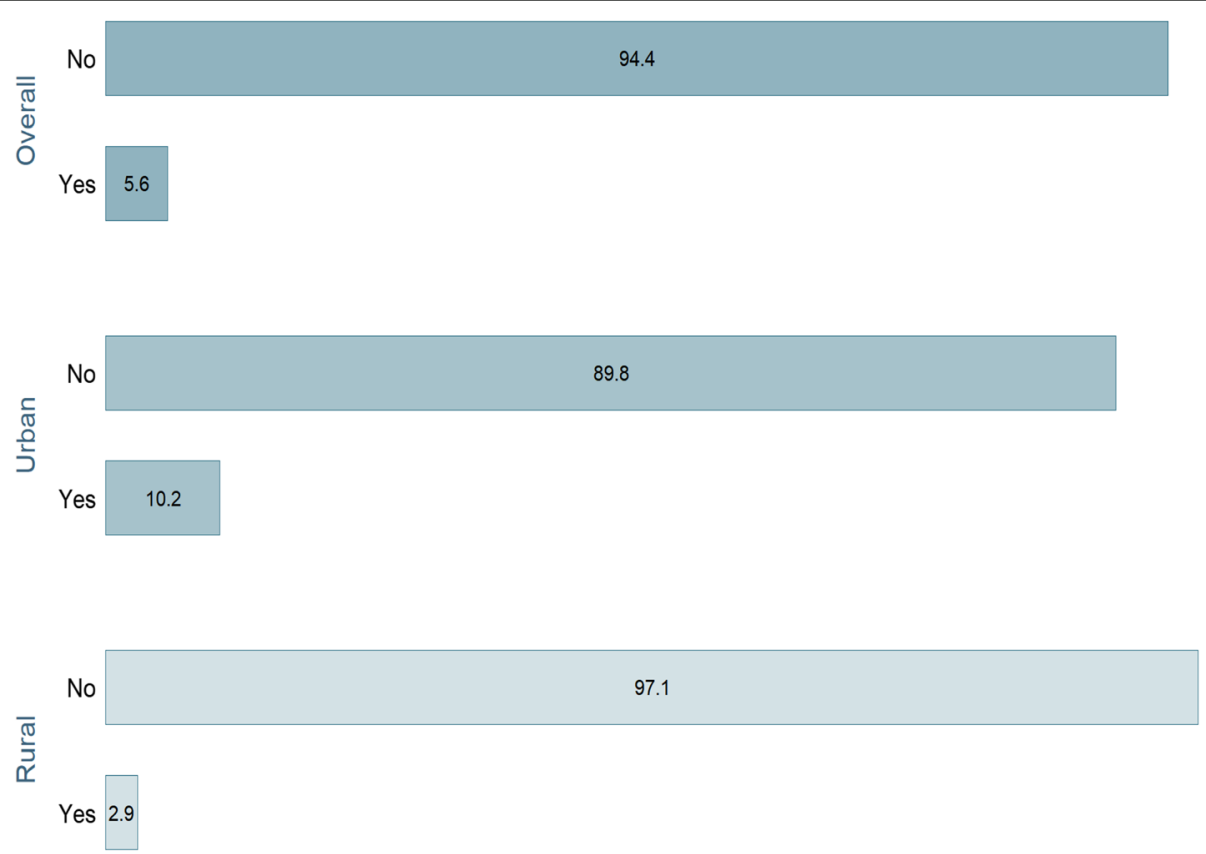

Fig. 2 Prevalence of C-section delivery 
Table 2 Predictors of using Facility Delivery Services in Mozambique

\begin{tabular}{|c|c|c|c|}
\hline & Pooled & Urban & Rural \\
\hline Age (15-19) & 1 & 1 & 1 \\
\hline $20-24$ & $\begin{array}{l}0.888 \\
{[0.704,1.120]}\end{array}$ & $\begin{array}{l}0.726 \\
{[0.411,1.281]}\end{array}$ & $\begin{array}{l}0.918 \\
{[0.710,1.186]}\end{array}$ \\
\hline $25-29$ & $\begin{array}{l}0.815 \\
{[0.645,1.030]}\end{array}$ & $\begin{array}{l}0.826 \\
{[0.463,1.473]}\end{array}$ & $\begin{array}{l}0.796 \\
{[0.614,1.031]}\end{array}$ \\
\hline $30-34$ & $\begin{array}{l}0.869 \\
{[0.678,1.115]}\end{array}$ & $\begin{array}{l}1.020 \\
{[0.542,1.922]}\end{array}$ & $\begin{array}{l}0.834 \\
{[0.634,1.096]}\end{array}$ \\
\hline $35-39$ & $\begin{array}{l}0.830 \\
{[0.631,1.093]}\end{array}$ & $\begin{array}{l}0.696 \\
{[0.359,1.352]}\end{array}$ & $\begin{array}{l}0.853 \\
{[0.629,1.157]}\end{array}$ \\
\hline $40-44$ & $\begin{array}{l}0.688^{*} \\
{[0.493,0.959]}\end{array}$ & $\begin{array}{l}0.671 \\
{[0.305,1.477]}\end{array}$ & $\begin{array}{l}0.685^{*} \\
{[0.473,0.992]}\end{array}$ \\
\hline $45-49$ & $\begin{array}{l}0.725 \\
{[0.472,1.115]}\end{array}$ & $\begin{array}{l}0.380 \\
{[0.130,1.114]}\end{array}$ & $\begin{array}{l}0.820 \\
{[0.513,1.311]}\end{array}$ \\
\hline Residency (Urban) & 1 & & \\
\hline Rural & $\begin{array}{l}0.527^{* * *} \\
{[0.440,0.630]}\end{array}$ & & \\
\hline Education (No Education) & 1 & 1 & 1 \\
\hline Primary & $\begin{array}{l}1.190^{*} \\
{[1.039,1.363]}\end{array}$ & $\begin{array}{l}1.163 \\
{[0.791,1.710]}\end{array}$ & $\begin{array}{l}1.160^{*} \\
{[1.002,1.343]}\end{array}$ \\
\hline Secondary & $\begin{array}{l}2.608^{* * *} \\
{[1.840,3.697]}\end{array}$ & $\begin{array}{l}1.860^{*} \\
{[1.030,3.358]}\end{array}$ & $\begin{array}{l}3.475^{* * *} \\
{[2.133,5.662]}\end{array}$ \\
\hline Higher & $\begin{array}{l}1.266^{*} \\
{[1.012,1.584]}\end{array}$ & $\begin{array}{l}1.643^{*} \\
{[1.123,2.403]}\end{array}$ & $\begin{array}{l}1.251 \\
{[0.896,1.748]}\end{array}$ \\
\hline Husband's education (No Education) & 1 & 1 & 1 \\
\hline Primary & $\begin{array}{l}0.979 \\
{[0.852,1.124]}\end{array}$ & $\begin{array}{l}0.953 \\
{[0.652,1.394]}\end{array}$ & $\begin{array}{l}0.980 \\
{[0.843,1.139]}\end{array}$ \\
\hline Secondary & $\begin{array}{l}1.737^{* * *} \\
{[1.370,2.203]}\end{array}$ & $\begin{array}{l}1.616 \\
{[1.000,2.613]}\end{array}$ & $\begin{array}{l}1.762^{* * *} \\
{[1.328,2.338]}\end{array}$ \\
\hline Higher & $\begin{array}{l}4.106 \\
{[0.553,30.47]}\end{array}$ & $\begin{array}{l}3.842 \\
{[0.497,29.68]}\end{array}$ & $\begin{array}{l}1.236^{*} \\
{[1.031,1.481]}\end{array}$ \\
\hline Employment (Not Working) & 1 & 1 & 1 \\
\hline Professional/Technical/Managerial & $\begin{array}{l}1.246^{*} \\
{[1.003,1.546]}\end{array}$ & $\begin{array}{l}1.189 \\
{[0.815,1.734]}\end{array}$ & $\begin{array}{l}1.302 \\
{[0.996,1.703]}\end{array}$ \\
\hline Agricultural - Self Employed & $\begin{array}{l}0.998 \\
{[0.872,1.142]}\end{array}$ & $\begin{array}{l}0.778 \\
{[0.534,1.134]}\end{array}$ & $\begin{array}{l}1.060 \\
{[0.916,1.227]}\end{array}$ \\
\hline Wealth quintile (Poorest) & 1 & 1 & 1 \\
\hline Poorer & $\begin{array}{l}1.181 \\
{[0.992,1.405]}\end{array}$ & $\begin{array}{l}1.128 \\
{[0.608,2.092]}\end{array}$ & $\begin{array}{l}1.174 \\
{[0.978,1.409]}\end{array}$ \\
\hline Middle & $\begin{array}{l}1.788^{* * *} \\
{[1.495,2.138]}\end{array}$ & $\begin{array}{l}1.601 \\
{[0.926,2.769]}\end{array}$ & $\begin{array}{l}1.774^{* * *} \\
{[1.465,2.147]}\end{array}$ \\
\hline Richer & $\begin{array}{l}2.718^{* * *} \\
{[2.217,3.333]}\end{array}$ & $\begin{array}{l}3.339^{* * *} \\
{[1.944,5.736]}\end{array}$ & $\begin{array}{l}2.452^{* * *} \\
{[1.954,3.077]}\end{array}$ \\
\hline Richest & $\begin{array}{l}4.898^{* * *} \\
{[3.547,6.764]}\end{array}$ & $\begin{array}{l}5.309^{* * *} \\
{[2.860,9.856]}\end{array}$ & $\begin{array}{l}6.064^{* * *} \\
{[3.542,10.38]}\end{array}$ \\
\hline Has media access (No) & 1 & 1 & 1 \\
\hline Yes & $\begin{array}{l}1.061 \\
{[0.931,1.209]}\end{array}$ & $\begin{array}{l}1.066 \\
{[0.739,1.537]}\end{array}$ & $\begin{array}{l}1.081 \\
{[0.938,1.246]}\end{array}$ \\
\hline Religion (Islam) & 1 & 1 & 1 \\
\hline Other & $\begin{array}{l}1.057 \\
{[0.924,1.210]}\end{array}$ & $\begin{array}{l}1.040 \\
{[0.739,1.463]}\end{array}$ & $\begin{array}{l}1.057 \\
{[0.912,1.226]}\end{array}$ \\
\hline Ethnicity (Emakhuwa) & 1 & 1 & 1 \\
\hline Portugais & $2.688^{* * *}$ & 1.636 & $4.556^{* *}$ \\
\hline
\end{tabular}


Table 2 Predictors of using Facility Delivery Services in Mozambique (Continued)

\begin{tabular}{|c|c|c|c|}
\hline & Pooled & Urban & Rural \\
\hline & {$[1.540,4.692]$} & {$[0.764,3.503]$} & {$[1.753,11.84]$} \\
\hline Xichangana & $\begin{array}{l}1.557^{* * *} \\
{[1.215,1.996]}\end{array}$ & $\begin{array}{l}0.931 \\
{[0.556,1.559]}\end{array}$ & $\begin{array}{l}1.894^{* * *} \\
{[1.414,2.535]}\end{array}$ \\
\hline Cisena & $\begin{array}{l}2.111^{* * *} \\
{[1.686,2.644]}\end{array}$ & $\begin{array}{l}5.427^{* * *} \\
{[2.450,12.02]}\end{array}$ & $\begin{array}{l}1.941^{* * *} \\
{[1.519,2.481]}\end{array}$ \\
\hline Elomwe & $\begin{array}{l}0.531^{* * *} \\
{[0.391,0.720]}\end{array}$ & $\begin{array}{l}0.246^{* * *} \\
{[0.115,0.524]}\end{array}$ & $\begin{array}{l}0.618^{* *} \\
{[0.443,0.864]}\end{array}$ \\
\hline Cindau & $\begin{array}{l}1.876^{* * *} \\
{[1.423,2.474]}\end{array}$ & $\begin{array}{l}0.933 \\
{[0.373,2.330]}\end{array}$ & $\begin{array}{l}2.073^{* * *} \\
{[1.548,2.775]}\end{array}$ \\
\hline Xitswa & $\begin{array}{l}0.949 \\
{[0.708,1.272]}\end{array}$ & $\begin{array}{l}0.723 \\
{[0.316,1.652]}\end{array}$ & $\begin{array}{l}1.010 \\
{[0.736,1.385]}\end{array}$ \\
\hline Other & $\begin{array}{l}1.440^{* * *} \\
{[1.215,1.708]}\end{array}$ & $\begin{array}{l}1.065 \\
{[0.693,1.638]}\end{array}$ & $\begin{array}{l}1.555^{* * *} \\
{[1.288,1.877]}\end{array}$ \\
\hline Parity (1-5) & 1 & 1 & 1 \\
\hline$>5$ & $\begin{array}{l}0.995 \\
{[0.830,1.192]}\end{array}$ & $\begin{array}{l}0.886 \\
{[0.562,1.395]}\end{array}$ & $\begin{array}{l}1.001 \\
{[0.820,1.223]}\end{array}$ \\
\hline Household head's sex (Male) & 1 & 1 & 1 \\
\hline Female & $\begin{array}{l}1.101 \\
{[0.965,1.257]}\end{array}$ & $\begin{array}{l}0.761 \\
{[0.561,1.033]}\end{array}$ & $\begin{array}{l}1.184^{*} \\
{[1.022,1.371]}\end{array}$ \\
\hline Child wantedness (Wanted Then) & 1 & 1 & 1 \\
\hline Wanted No More & $\begin{array}{l}1.059 \\
{[0.881,1.273]}\end{array}$ & $\begin{array}{l}1.383 \\
{[0.947,2.020]}\end{array}$ & $\begin{array}{l}0.984 \\
{[0.795,1.219]}\end{array}$ \\
\hline Antenatal Visits $(<4)$ & 1 & 1 & 1 \\
\hline 4 or more & $\begin{array}{l}1.340^{* * *} \\
{[1.188,1.512]}\end{array}$ & $\begin{array}{l}1.655^{* * *} \\
{[1.235,2.218]}\end{array}$ & $\begin{array}{l}1.265^{* * *} \\
{[1.108,1.445]}\end{array}$ \\
\hline Pseudo $R^{2}$ & 0.184 & 0.190 & 0.199 \\
\hline
\end{tabular}

Exponentiated coefficients; $95 \%$ confidence intervals in brackets

${ }^{*} p<0.05,{ }^{* *} p<0.01,{ }^{* * *} p<0.001$

in the use of c-section services as well, with the prevalence being $10.2 \%$ in the urban and $2.9 \%$ in the rural areas. While prevalence of c-sections in urban areas aligned with the World Health Organization recommended cut-off of $10-15 \%$, that of the rural areas was remarkably low [29]. Better availability of the medical infrastructure and awareness about the use of c-section services have spurred its global prevalence ( 21\%), which is far higher than the African average of about $5 \%$ [30]. Lower use of C-section services can stem from various causes such as affordability and awareness; these should be investigated and addressed to reduce inequality.

There were sociodemographic disparities in the utilization of facility delivery and c-section services. Women with higher education were significantly more likely to use facility delivery services compared with those who had no education. The positive effect of education on health service seeking behaviour can function in two ways. Firstly, educated women are more likely to be aware of the danger signs of pregnancy and the risk factors of pregnancy complications; hence, they are more likely to avail themselves of the services [31, 32].
Secondly, educated women are expected to be more financially empowered and have better decision-making autonomy [33]. These factors can enable them to make better use of the services which would otherwise be unaffordable or inaccessible to them. Furthermore, the findings revealed that women living in households with higher wealth status had higher odds of using both facility delivery and c-section services. Financial well-being is a predictor of general health status as well as positive healthcare-seeking behaviour. Therefore, removing financial constraints in accessing child-birth services should be considered an important priority in Mozambique.

Women's ethnic background was an important predictor of using facility delivery services as well. The racial/ethnic differences in maternal health service use and health outcomes have been a subject of growing interest in many countries [34-37]. The underlying factors behind this difference generally consist of lower socioeconomic status and perception of health and healthcare $[29,38]$. In some cultures, delivering at home may be considered normal or more culturally appropriate, thereby making the practice more common even when 
Table 3 Predictors of using Caesarean Section Services in Mozambique

\begin{tabular}{|c|c|c|c|}
\hline & Pooled & Urban & Rural \\
\hline Age (15-19) & 1 & 1 & 1 \\
\hline $20-24$ & $\begin{array}{l}0.662^{*} \\
{[0.441,0.993]}\end{array}$ & $\begin{array}{l}0.546^{*} \\
{[0.325,0.917]}\end{array}$ & $\begin{array}{l}0.924 \\
{[0.474,1.800]}\end{array}$ \\
\hline $25-29$ & $\begin{array}{l}0.739 \\
{[0.491,1.110]}\end{array}$ & $\begin{array}{l}0.592^{*} \\
{[0.352,0.995]}\end{array}$ & $\begin{array}{l}1.079 \\
{[0.549,2.123]}\end{array}$ \\
\hline $30-34$ & $\begin{array}{l}0.755 \\
{[0.485,1.175]}\end{array}$ & $\begin{array}{l}0.680 \\
{[0.389,1.190]}\end{array}$ & $\begin{array}{l}0.877 \\
{[0.412,1.868]}\end{array}$ \\
\hline $35-39$ & $\begin{array}{l}1.044 \\
{[0.648,1.681]}\end{array}$ & $\begin{array}{l}0.854 \\
{[0.465,1.569]}\end{array}$ & $\begin{array}{l}1.504 \\
{[0.681,3.320]}\end{array}$ \\
\hline $40-44$ & $\begin{array}{l}1.463 \\
{[0.794,2.695]}\end{array}$ & $\begin{array}{l}1.282 \\
{[0.594,2.770]}\end{array}$ & $\begin{array}{l}1.717 \\
{[0.612,4.818]}\end{array}$ \\
\hline $45-49$ & $\begin{array}{l}1.366 \\
{[0.519,3.596]}\end{array}$ & $\begin{array}{l}0.858 \\
{[0.168,4.387]}\end{array}$ & $\begin{array}{l}2.057 \\
{[0.575,7.356]}\end{array}$ \\
\hline Residency (Urban) & 1 & & \\
\hline Rural & $\begin{array}{l}0.546^{* * *} \\
{[0.396,0.752]}\end{array}$ & & \\
\hline Education (No Education) & 1 & 1 & 1 \\
\hline Primary & $\begin{array}{l}1.327 \\
{[0.926,1.902]}\end{array}$ & $\begin{array}{l}0.969 \\
{[0.545,1.725]}\end{array}$ & $\begin{array}{l}1.541 \\
{[0.970,2.447]}\end{array}$ \\
\hline Secondary & $\begin{array}{l}2.114^{* *} \\
{[1.335,3.347]}\end{array}$ & $\begin{array}{l}1.461 \\
{[0.766,2.786]}\end{array}$ & $\begin{array}{l}2.979^{* *} \\
{[1.425,6.226]}\end{array}$ \\
\hline Higher & $\begin{array}{l}4.568^{* * *} \\
{[2.105,9.912]}\end{array}$ & $\begin{array}{l}3.269^{*} \\
{[1.327,8.050]}\end{array}$ & $\begin{array}{l}1.601 \\
{[0.926,2.769]}\end{array}$ \\
\hline Husband's education (No Education) & 1 & 1 & 1 \\
\hline Primary & $\begin{array}{l}0.727 \\
{[0.528,1.003]}\end{array}$ & $\begin{array}{l}0.751 \\
{[0.466,1.212]}\end{array}$ & $\begin{array}{l}0.697 \\
{[0.448,1.085]}\end{array}$ \\
\hline Secondary & $\begin{array}{l}0.779 \\
{[0.532,1.140]}\end{array}$ & $\begin{array}{l}0.777 \\
{[0.469,1.287]}\end{array}$ & $\begin{array}{l}0.825 \\
{[0.441,1.544]}\end{array}$ \\
\hline Higher & $\begin{array}{l}1.280 \\
{[0.701,2.335]}\end{array}$ & $\begin{array}{l}1.430 \\
{[0.722,2.835]}\end{array}$ & $\begin{array}{l}1.020 \\
{[0.641,1.722]}\end{array}$ \\
\hline Employment (Not Working) & 1 & 1 & 1 \\
\hline Professional/Technical/Managerial & $\begin{array}{l}1.210 \\
{[0.921,1.588]}\end{array}$ & $\begin{array}{l}1.324 \\
{[0.966,1.814]}\end{array}$ & $\begin{array}{l}0.864 \\
{[0.472,1.581]}\end{array}$ \\
\hline Agricultural - Self Employed & $\begin{array}{l}0.696^{*} \\
{[0.488,0.993]}\end{array}$ & $\begin{array}{l}0.675 \\
{[0.366,1.244]}\end{array}$ & $\begin{array}{l}0.654 \\
{[0.415,1.031]}\end{array}$ \\
\hline Wealth quintile (Poorest) & 1 & 1 & 1 \\
\hline Poorer & $\begin{array}{l}1.368 \\
{[0.749,2.498]}\end{array}$ & $\begin{array}{l}1.101 \\
{[0.909,1.334]}\end{array}$ & $\begin{array}{l}1.767 \\
{[0.903,3.457]}\end{array}$ \\
\hline Middle & $\begin{array}{l}1.548 \\
{[0.873,2.745]}\end{array}$ & $\begin{array}{l}1.660 \\
{[0.579,4.757]}\end{array}$ & $\begin{array}{l}1.289 \\
{[0.635,2.617]}\end{array}$ \\
\hline Richer & $\begin{array}{l}1.507 \\
{[0.850,2.670]}\end{array}$ & $\begin{array}{l}0.821 \\
{[0.289,2.330]}\end{array}$ & $\begin{array}{l}1.791 \\
{[0.875,3.665]}\end{array}$ \\
\hline Richest & $\begin{array}{l}2.077^{*} \\
{[1.101,3.919]}\end{array}$ & $\begin{array}{l}1.336 \\
{[0.469,3.808]}\end{array}$ & $\begin{array}{l}2.277 \\
{[0.904,5.737]}\end{array}$ \\
\hline Has media access (No) & 1 & 1 & 1 \\
\hline Yes & $\begin{array}{l}0.926 \\
{[0.678,1.264]}\end{array}$ & $\begin{array}{l}0.921 \\
{[0.564,1.505]}\end{array}$ & $\begin{array}{l}0.956 \\
{[0.633,1.446]}\end{array}$ \\
\hline Religion (Islam) & 1 & 1 & 1 \\
\hline Other & $\begin{array}{l}0.812 \\
{[0.610,1.080]}\end{array}$ & $\begin{array}{l}0.993 \\
{[0.693,1.422]}\end{array}$ & $\begin{array}{l}0.637 \\
{[0.392,1.036]}\end{array}$ \\
\hline Ethnicity (Emakhuwa) & 1 & 1 & 1 \\
\hline Portugais & 1.346 & 1.742 & 1.612 \\
\hline
\end{tabular}


Table 3 Predictors of using Caesarean Section Services in Mozambique (Continued)

\begin{tabular}{|c|c|c|c|}
\hline & Pooled & Urban & Rural \\
\hline & {$[0.815,2.224]$} & {$[0.910,3.336]$} & {$[0.570,4.561]$} \\
\hline Xichangana & $\begin{array}{l}1.243 \\
{[0.775,1.993]}\end{array}$ & $\begin{array}{l}1.687 \\
{[0.892,3.190]}\end{array}$ & $\begin{array}{l}0.821 \\
{[0.377,1.788]}\end{array}$ \\
\hline Cisena & $\begin{array}{l}1.325 \\
{[0.775,2.265]}\end{array}$ & $\begin{array}{l}1.759 \\
{[0.835,3.706]}\end{array}$ & $\begin{array}{l}1.085 \\
{[0.490,2.405]}\end{array}$ \\
\hline Elomwe & $\begin{array}{l}0.487 \\
{[0.146,1.628]}\end{array}$ & $\begin{array}{l}0.563 \\
{[0.0712,4.457]}\end{array}$ & $\begin{array}{l}0.405 \\
{[0.0906,1.809]}\end{array}$ \\
\hline Cindau & $\begin{array}{l}1.647 \\
{[0.908,2.987]}\end{array}$ & $\begin{array}{l}2.669^{*} \\
{[1.048,6.801]}\end{array}$ & $\begin{array}{l}1.254 \\
{[0.564,2.790]}\end{array}$ \\
\hline Xitswa & $\begin{array}{l}1.817 \\
{[0.989,3.338]}\end{array}$ & $\begin{array}{l}2.443 \\
{[0.986,6.052]}\end{array}$ & $\begin{array}{l}1.508 \\
{[0.653,3.480]}\end{array}$ \\
\hline Other & $\begin{array}{l}1.127 \\
{[0.731,1.738]}\end{array}$ & $\begin{array}{l}1.481 \\
{[0.793,2.768]}\end{array}$ & $\begin{array}{l}0.905 \\
{[0.490,1.674]}\end{array}$ \\
\hline Parity (1-5) & 1 & 1 & 1 \\
\hline$>5$ & $\begin{array}{l}0.627^{*} \\
{[0.407,0.964]}\end{array}$ & $\begin{array}{l}0.627 \\
{[0.339,1.157]}\end{array}$ & $\begin{array}{l}0.651 \\
{[0.346,1.224]}\end{array}$ \\
\hline Household head's sex (Male) & 1 & 1 & 1 \\
\hline Female & $\begin{array}{l}1.122 \\
{[0.886,1.422]}\end{array}$ & $\begin{array}{l}1.101 \\
{[0.814,1.489]}\end{array}$ & $\begin{array}{l}1.109 \\
{[0.752,1.637]}\end{array}$ \\
\hline Child wantedness (Wanted Then) & 1 & 1 & 1 \\
\hline Wanted No More & $\begin{array}{l}0.930 \\
{[0.709,1.222]}\end{array}$ & $\begin{array}{l}0.821 \\
{[0.594,1.134]}\end{array}$ & $\begin{array}{l}1.379 \\
{[0.836,2.276]}\end{array}$ \\
\hline Antenatal Visits $(<4)$ & 1 & 1 & 1 \\
\hline 4 or more & $\begin{array}{l}1.393^{*} \\
{[1.077,1.800]}\end{array}$ & $\begin{array}{l}1.270 \\
{[0.910,1.771]}\end{array}$ & $\begin{array}{l}1.570^{*} \\
{[1.042,2.365]}\end{array}$ \\
\hline Pseudo $R^{2}$ & 0.181 & 0.173 & 0.160 \\
\hline
\end{tabular}

Exponentiated coefficients; $95 \%$ confidence intervals in brackets

${ }^{*} p<0.05,{ }^{* *} p<0.01,{ }^{* * *} p<0.001$

professional birthing services are available [38]. Sociocultural factors that shape healthcare-seeking behaviour are inherently more challenging to address and require special strategies such as replacing the existing norms and beliefs with the new ones through community education (role play) programs $[39,40]$. It is important to design context-specific and locally-relevant interventions that increase uptake, avoiding the appearance of being culturally invasive [41].

Lastly, we found an association between the use of ANC visits and professional birthing services. ANC programs are generally educational and serve as the preparatory stage for safe delivery and successful termination of a pregnancy. Women who contact careproviders during the ANC stage are more likely to learn about and plan the place of delivery [42]. Promoting the use of ANC services appears to be an important driver for scaling up facility delivery programs.

This study provided relevant insights regarding the prevalence and use of facility delivery and c-section services use in a nationally-representative sample in Mozambique. Findings contribute to an existing gap in the literature and generate potential areas of investigation for future research. Significant differences were found in the use of facility delivery services among certain ethnicities. While these findings provide a general idea regarding the nature of ethnic inequality, it did not allow the investigation of underlying mechanisms contributing to the trend. Further qualitative studies should be carried out to explore the sources of disparity and potential areas of intervention.

Several limitations should be noted for this study. The data were cross-sectional and hence no causality can be inferred from the associations. Authors used a secondary database and, therefore, have no influence over the selection and measurement of the variables. As the data were self-reported, the chances of recall and reporting bias cannot be ignored. The factors that influence healthcare service utilization are diverse and multifaceted, but the choice of the explanatory factors was limited to what existed in the Mozambique 2011 DHS survey. Factors such as geographical distance, transportation facilities, quality of services in local healthcare settings, availability of female care provider have also been found to be important in primary studies; we were not able to adjust for these factors in the current analysis. 
The use of C-section services is also a complex outcome that can be driven by various personal and medical factors; no such data were collected in the Mozambique DHS. The survey was conducted in 2011, and therefore may not represent the current scenario. Lastly, due to the nature of the survey and analyses, causality cannot be inferred for the relationship between the outcomes (place of delivery and use of c-section services) and associated explanatory factors.

\section{Conclusion}

This was a secondary analysis of the 2011 Mozambique Demographic and Health Survey data on the use of professional childbirth services among community dwelling women. The findings indicate that approximately $30 \%$ of the women were not using health facility delivery services, with the difference denoting a considerable urbanrural gap. Significant differences were also observed for women's education, household wealth, and ethnic background.

\section{Abbreviations}

ANC: Antenatal Care; CS: C-Section; DHS: Demographic and Health Surveys; MCM: Maternal and child mortality; TBAs: Traditional Birth Attendants; SBAs: Skilled birth attendants; WHO: World Health Organization

\section{Acknowledgements}

The authors thank the MEASURE DHS project for their support and for free access to the original data.

\section{Authors' contributors}

SY and GB designed the study and drafted the initial manuscript. DIW, GS and AA contributed to the literature review, data analysis and interpretation of the original findings. SY had final responsibility to submit for publication. All authors read and amended drafts of the paper and approved the final version.

\section{Funding}

The authors have no support or funding to report.

\section{Availability of data and materials}

Data for this study were sourced from Demographic and Health surveys (DHS) and available here: http://dhsprogram.com/data/available-datasets.cfm.

\section{Ethics approval and consent to participate}

Ethics approval for this study was not required since the data is secondary and is available in the public domain. More details regarding DHS data and ethical standards are available at: http://goo.gl/ny8T6X.

\section{Consent for publication}

No consent to publish was needed for this study as we did not use any details, images or videos related to individual participants. In addition, data used is available in the public domain.

\section{Competing interests}

Sanni Yaya is Editor-in-Chief of this journal.

\section{Author details}

${ }^{1}$ School of International Development and Global Studies, Faculty of Social Sciences, University of Ottawa, 120 University Private, Ottawa, ON K1N 6N5, Canada. ${ }^{2}$ The George Institute for Global Health, University of Oxford, Oxford, UK. ${ }^{3}$ Faculty of Health Sciences, University of Ottawa, Ottawa, Canada. ${ }^{4}$ Bloomberg School of Public Health, Johns Hopkins University, Baltimore, MD, USA. ${ }^{5}$ Department of Reproductive Health and Health Services
Management, School of Public Health, Addis Ababa University, Addis Ababa, Ethiopia.

Received: 7 January 2020 Accepted: 8 April 2020

Published online: 16 April 2020

\section{References}

1. Hanlon J,Cunguara B. Poverty is not being reduced in Mozambique. LSE Crisis States Research Centre. 2010. Available at https://oro.open.ac.uk/232 70/1/WP74.2.pdf. Accessed on March 28, 2020.

2. Heltberg R, Simler K, Tarp F. Public spending and poverty in Mozambique International Food Policy Research Institute. FCND Discusson paper \# 167. Available at https://ageconsearch.umn.edu/record/16462/files/fc030167.pdf. Accessed on March 28, 2020.

3. Addison T, de Sousa C. Mozambique: Economic Reform and Reconstruction. In: McGillivray M., Morrissey O. (eds) Evaluating Economic Liberalization. Case-Studies in Economic Development. 1999. Palgrave Macmillan, London.

4. World Bank. "Strong but not broadly shared growth". Mozambique _ poverty assessment. Poverty and Equity Global Practice Africa Region Available at http://documents.worldbank.org/curated/en/24856154116504 0969/pdf/Mozambique-Poverty-Assessment-Strong-But-Not-Broadly-SharedGrowth.pdf. Accessed on March 28, 2020.

5. United Nations Population Division. Mozambique: Data source: United Nations World Population Prospects. Available at https://population.un.org/ wpp/. Accessed on March 28, 2020.

6. Augusto O, Keyes EE, Madede T, Abacassamo F, de la Corte P, Chilundo B, Bailey PE. Progress in Mozambique: changes in the availability, use, and quality of emergency obstetric and newborn care between 2007 and 2012. PLoS One. 2018 Jul 18;13(7):e0199883. https://doi.org/10.1371/journal.pone. 0199883.

7. Population Reference Bureau Mozambique's High Rate of Maternal Mortality -. [cited 28 May 2019]. Available: https://www.prb.org/ mozambiqueshighrateofmaternalmortality.

8. Darmstadt GL, Lee AC, Cousens S, Sibley L, Bhutta ZA, Donnay F, et al. 60 million non-facility births: who can deliver in community settings to reduce intrapartum-related deaths? Int J Gynaecol Obstet. 2009;107:S89-112. https://doi.org/10.1016/j.ijgo.2009.07.010.

9. Sebghati M, Chandraharan E. An update on the risk factors for and management of obstetric haemorrhage. Womens Health (Lond) 2017 Aug; 13(2):34-40. doi: https://doi.org/10.1177/1745505717716860. Epub 2017 Jul 6.

10. Geller SE, Koch AR, Garland CE, MacDonald EJ, Storey F, Lawton B. A global view of severe maternal morbidity: moving beyond maternal mortality. Reprod Health. 2018;15. https://doi.org/10.1186/s12978-018-0527-2.

11. Girum T, Wasie A. Correlates of maternal mortality in developing countries: an ecological study in 82 countries. Matern Health Neonatol Perinatol. 2017; 3. https://doi.org/10.1186/s40748-017-0059-8.

12. Banerjee J, Kaur C, Ramaiah S, Roy R, Aladangady N. Factors influencing the uptake of neonatal bereavement support services - findings from two tertiary neonatal centres in the UK. BMC Palliat Care. 2016 Jun 29;15:54. https://doi.org/10.1186/s12904-016-0126-3.

13. Kumar S, Gruskin S, Khosla R, Narasimhan M. Human rights and the sexual and reproductive health of women living with HIV - a literature review. J Int AIDS Soc. 2015;18. https://doi.org/10.7448/IAS.18.6.20290.

14. Khosla R, Van Belle N, Temmerman M. Advancing the sexual and reproductive health and human rights of women living with HIV: a review of UN, regional and national human rights norms and standards. J Int AIDS Soc. 2015 ;18(Suppl 5):20280. doi: https://doi.org/10.7448/IAS.18.6.20280. PMID: 26643455; PMCID: PMC4672403.

15. Mylonas I, Friese K. Indications for and risks of elective cesarean section. Dtsch Arztebl Int. 2015;112:489-95. https://doi.org/10.3238/arztebl.2015.0489.

16. Keag OE, Norman JE, Stock SJ. Long-term risks and benefits associated with cesarean delivery for mother, baby, and subsequent pregnancies: systematic review and meta-analysis. PLoS Med. 2018;15. https://doi.org/10.1371/ journal.pmed.1002494.

17. The Business Year. The Right Policy - [cited 10 Dec 2019]. Available: https:// www.thebusinessyear.com/mozambique-2015/the-right-policy/review.

18. Boah M, Mahama AB, Ayamga EA. They receive antenatal care in health facilities, yet do not deliver there: predictors of health facility delivery by women in rural Ghana. BMC Pregnancy Childbirth. 2018;18. https://doi.org/ 10.1186/s12884-018-1749-6. 
19. Atnafu A, Otto $\mathrm{K}$, Herbst $\mathrm{CH}$. The role of mHealth intervention on maternal and child health service delivery: findings from a randomized controlled field trial in rural Ethiopia. Mhealth. 2017;3. https://doi.org/10.21037/mhealth. 2017.08.04.

20. Kerber KJ, de Graft-Johnson JE, Bhutta ZA, Okong P, Starrs A, Lawn JE. Continuum of care for maternal, newborn, and child health: from slogan to service delivery. Lancet. 2007;370:1358-69. https://doi.org/10.1016/S01406736(07)61578-5.

21. Konkor I, Sano Y, Antabe R, Kansanga M, Luginaah I. Exposure to mass media family planning messages among post-delivery women in Nigeria: testing the structural influence model of health communication. Eur J Contracept Reprod Health Care 2019;0: 1-6. doi:https://doi.org/10.1080/ 13625187.2018.1563679.

22. Gouda HN, Hodge A, Bermejo R III, Zeck W, Jimenez-Soto E. The Impact of Healthcare Insurance on the Utilisation of Facility-Based Delivery for Childbirth in the Philippines. [cited 17 Feb 2019]. Available: https://journals. plos.org/plosone/article?id=10.1371/journal.pone.0167268.

23. Tesfaye G, Chojenta C, Smith R, Loxton D. Application of the AndersenNewman model of health care utilization to understand antenatal care use in Kersa District. Eastern Ethiopia PLOS ONE. 2018;13:e0208729. https://doi. org/10.1371/journal.pone.0208729.

24. Sakshaug JW, West BT. Important considerations when analyzing health survey data collected using a complex sample design. Am J Public Health. 2014;104:15-6. https://doi.org/10.2105/AJPH.2013.301515.

25. Yaya S, Bishwajit G, Shah V. Wealth, education and urban-rural inequality and maternal healthcare service usage in Malawi. BMJ Glob Health. 2016;1. https://doi.org/10.1136/bmjgh-2016-000085.

26. Ousman SK, Mdala I, Thorsen VC, Sundby J, Magnus JH. Social determinants of antenatal care service use in Ethiopia: changes over a 15-year span. Front Public Health. 2019;7. https://doi.org/10.3389/fpubh.2019.00161.

27. Arsenault C, Jordan K, Lee D, Dinsa G, Manzi F, Marchant T, et al. Equity in antenatal care quality: an analysis of 91 national household surveys. Lancet Glob Health. 2018;6:e1186-95. https://doi.org/10.1016/ S2214-109X(18)30389-9.

28. Adewuyi EO, Auta A, Khanal V, Bamidele OD, Akuoko CP, Adefemi K, et al. Prevalence and factors associated with underutilization of antenatal care services in Nigeria: A comparative study of rural and urban residences based on the 2013 Nigeria demographic and health survey. PLoS One 2018; 13. doi:https://doi.org/10.1371/journal.pone.0197324.

29. Betran AP, Torloni MR, Zhang J, Ye J, Mikolajczyk R, Deneux-Tharaux C, et al. What is the optimal rate of caesarean section at population level? Reprod Health. 2015;12:57. https://doi.org/10.1186/s12978-015-0043-6.

30. The Lancet. Stemming the global caesarean section epidemic. The Lancet. 2018;392: 1279. doi:https://doi.org/10.1016/S0140-6736(18)32394-8.

31. Ghose B, Feng D, Tang S, Yaya S, He Z, Udenigwe O, et al. Women's decision-making autonomy and utilisation of maternal healthcare services: results from the Bangladesh demographic and health survey. BMJ Open. 2017;7:e017142. https://doi.org/10.1136/bmjopen-2017-017142.

32. Al-Ateeq MA, Al-Rusaiess AA. Health education during antenatal care: the need for more. Int J Women's Health. 2015;7:239-42. https://doi.org/10. 2147/IJWH.S75164.

33. Yaya S, Bishwajit G, Ekholuenetale M, Shah V. Awareness and utilization of community clinic services among women in rural areas in Bangladesh: a cross-sectional study. PLoS One. 2017;12:e0187303. https://doi.org/10.1371/ journal.pone.0187303.

34. Bryant AS, Worjoloh A, Caughey AB, Washington AE. Racial/ethnic disparities in obstetrical outcomes and care: prevalence and determinants. Am J Obstet Gynecol. 2010;202:335-43. https://doi.org/10.1016/j.ajog.2009.10.864.

35. Hong J, Bishop-Fitzpatrick L, Smith L, Greenberg JS, Mailick MR. Factors associated with subjective quality of life of adults with autism Spectrum disorder: self-report vs. Maternal Reports J Autism Dev Disord. 2016;46:136878. https://doi.org/10.1007/s10803-015-2678-0.

36. Adu J, Tenkorang E, Banchani E, Allison J, Mulay S. The effects of individual and community-level factors on maternal health outcomes in Ghana. PLoS One. 2018;13:e0207942. https://doi.org/10.1371/journal.pone.0207942.

37. Wu Y, Zhou H, Wang Q, Cao M, Medina A, Rozelle S. Use of maternal health services among women in the ethnic rural areas of western China. BMC Health Serv Res. 2019;19:179. https://doi.org/10.1186/s12913-019-3996-2.

38. Yaya S, Bishwajit G, Uthman OA, Amouzou A. Why some women fail to give birth at health facilities: a comparative study between Ethiopia and Nigeria. PLoS One. 2018;13:e0196896. https://doi.org/10.1371/journal.pone.0196896.
39. The importance of values education in today's society. In: Iberdrola [Internet]. [cited 10 Dec 2019]. Available: https://www.iberdrola.com/talent/ value-education.

40. Andermann A. Taking action on the social determinants of health in clinical practice: a framework for health professionals. CMAJ. 2016;188:E474-83. https://doi.org/10.1503/cmaj.160177.

41. Manfredo MJ, Bruskotter JT, Teel TL, Fulton D, Schwartz SH, Arlinghaus R, et al. Why social values cannot be changed for the sake of conservation. Conserv Biol. 2017;31:772-80. https://doi.org/10.1111/cobi.12855.

42. Yaya S, Bishwajit G, Ekholuenetale M. Factors associated with the utilization of institutional delivery services in Bangladesh. PLoS One. 2017;12. https:// doi.org/10.1371/journal.pone.0171573.

\section{Publisher's Note}

Springer Nature remains neutral with regard to jurisdictional claims in published maps and institutional affiliations.
Ready to submit your research? Choose BMC and benefit from:

- fast, convenient online submission

- thorough peer review by experienced researchers in your field

- rapid publication on acceptance

- support for research data, including large and complex data types

- gold Open Access which fosters wider collaboration and increased citations

- maximum visibility for your research: over $100 \mathrm{M}$ website views per year

At BMC, research is always in progress.

Learn more biomedcentral.com/submissions 